Article

\title{
Genetic Identification and Drug-Resistance Characterization of Mycobacterium tuberculosis Using a Portable Sequencing Device. A Pilot Study
}

\author{
Jorge Cervantes ${ }^{1, *(\mathbb{D}}$, Noemí Yokobori ${ }^{2}{ }^{\mathbb{D}}$ and Bo-Young Hong ${ }^{3}$ \\ 1 Paul L. Foster School of Medicine, Texas Tech University Health Sciences Center, El Paso, TX 79905, USA \\ 2 Servicio de Micobacterias, Instituto Nacional de Enfermedades Infecciosas (INEI)-ANLIS and CONICET, \\ Buenos Aires C1282AFF, Argentina; kaoru.noemi@gmail.com \\ 3 The Jackson Laboratory for Genomic Medicine, Farmington, CT 06032, USA; auvers@gmail.com \\ * Correspondence: jorge.cervantes@ttuhsc.edu; Tel.: +1-915-215-4672
}

Received: 29 June 2020; Accepted: 26 August 2020; Published: 27 August 2020

\begin{abstract}
Clinical management of tuberculosis (TB) in endemic areas is often challenged by a lack of resources including laboratories for Mycobacterium tuberculosis (Mtb) culture. Traditional phenotypic drug susceptibility testing for Mtb is costly and time consuming, while PCR-based methods are limited to selected target loci. We herein utilized a portable, USB-powered, long-read sequencing instrument (MinION), to investigate Mtb genomic DNA from clinical isolates to determine the presence of anti-TB drug-resistance conferring mutations. Data analysis platform EPI2ME and antibiotic-resistance analysis using the real time ARMA workflow, identified Mtb species as well as extensive resistance gene profiles. The approach was highly sensitive, being able to detect almost all described drug resistance conferring mutations based on previous whole genome sequencing analysis. Our findings are supportive of the practical use of this system as a suitable method for the detection of antimicrobial resistance genes, and effective in providing Mtb genomic information. Future improvements in the error rate through statistical analysis, drug resistance prediction algorithms and reference databases would make this a platform suited for the clinical setting. The small size, relatively inexpensive cost of the device, as well as its rapid and simple library preparation protocol and analysis, make it an attractive option for settings with limited laboratory infrastructure.
\end{abstract}

Keywords: next-generation sequencing; MinION; tuberculosis; drug resistance

\section{Introduction}

Tuberculosis (TB) is the number one cause of human death due to an infectious disease, with 1.7 million deaths per year worldwide [1]. The causative agents of TB are a group of closely related bacteria known as the Mycobacterium tuberculosis (Mtb) complex (MTBC), which has been thought to have low DNA sequence diversity [2]. This limited diversity, however, is influenced by selective pressures and background selection [2]. Various human-adapted MTBC variants are known to differ in virulence, progression of disease and transmission potential.

TB surveillance of highly-virulent and multi-drug resistant (MDR) strains is paramount for adequate diagnosis and treatment $[1,3]$. Traditional phenotypic drug susceptibility testing (DST) through culture-based methods has multiple caveats, amongst them being that TB culturing can take days to weeks [4]. To reduce the time to obtain test results, alternative methods like real-time PCR-based Xpert MTB/RIF testing have been recommended by the World Health Organization [5]. These methods, however, are unable to detect drug-resistance mutations outside of the selected target loci [6], or they can produce false positive results [7]. In addition, clinical management where TB risk is high is often challenged by a lack of resources such as facilities for chest X-rays or laboratories for Mtb isolation 
and culture. To address these challenges, a whole-genome sequencing (WGS) approach can generate antibiotic susceptibility profiles, detect MDR-TB, and discover other MTB virulence factors [3,4]. This method, however, is also limited by resources, hospital-laboratory infrastructure and personnel training in bioinformatic analysis. A hybridization-based system (reverse line probe assay) has been recently proposed as an alternative in cost to WGS, but since this methodology is based on hybridization, it is also limited to the genomic region of Mtb examined [8]. Furthermore, although the cost per sample is much less than for other assays, it still requires laboratory equipment.

Development of a diagnostic assay that can be used at the point of care to rapidly and accurately diagnose TB and to include multidrug-resistant tuberculosis (MDR-TB) or extensively drug-resistant tuberculosis (XDR-TB) should be given a high priority. MDR-TB characterization typically requires costly machinery and handling in a specialized reference laboratory, not to mention the time required for shipping and processing the sample. A portable sequencing system that could be taken to the field, would not only reduce the cost of TB testing, but will also speed up the diagnoses. A rapid direct sample sequencing device would significantly reduce the time to obtain test results.

The MinION -Oxford Nanopore Technologies Limited (ONT), is a pocket sized $(10 \times 3 \times 2 \mathrm{~cm})$, portable, USB-powered, long-read sequencing instrument [9]. Among the existing sequencing platforms, it has the potential to be the best suited method to investigate the chain of transmission of TB and to determine the susceptibility of anti-TB drugs in the near future. This platform is particularly useful in remote settings or with limited infrastructure [9]. A careful evaluation of MinION as a potential methodology for the surveillance of TB was first reported in 2017 [10]. In this investigation, authors used both Illumina and ONT platforms for the diagnosis of Mtb infection. Utilization of the MinION in this study was conducted only with simulated Mtb infection using Ziehl-Neelsen (ZN)-negative sputum DNA combined with Mycobacterium bovis BCG strain DNA, not direct sputum sample. Despite the advantage of a portable sequencer in MDR-TB testing, so far there is no peer-reviewed published protocol of ONT-WGS based, rapid MDR-TB testing of patient sputum samples. It is unknown if this portable DNA sequencing system would be effective in providing information on Mtb genotype, drug-susceptibility in a sputum sample.

In this pilot study, we evaluated the performance of this portable sequence system for Mtb species identification and detection of genes related to drug resistance, as a means of MDR-TB testing in a diverse set of samples, including DNA isolated from sputum samples and from clinical microbiological isolates.

\section{Results}

\subsection{Identification of Mtb in Clinical Microbiological Isolates and Sputum Samples}

Upon sequencing of a set of DNA obtained from various sources, utilizing the What's In My Pot (WIMP) app [11] we were able to identify Mtb in all samples evaluated in this study. It was evident that DNA extracted directly from sputa yielded a great number of reads for human DNA (Table 1), with only a few reads for Mtb. In contrast, the presence of reads assigned to Homo sapiens in clinical isolates was minimal, and absent in the commercial Mtb genomic DNA.

\subsection{Identification of Drug Resistance Conferring Mutations in DNA}

We evaluated molecular genome-based drug resistance mutation analysis by sequencing DNA samples using a portable, long-read sequencing platform. Sequenced and analyzed data from Mtb culture isolates and commercially available Mtb genomic DNA showed numerous drug resistance-conferring mutations (Table 2 and Appendix A, Appendix B, Appendix C, Appendix D, Appendix E, Appendix F, Appendix G).

Comparison of the sequencing results of Mtb DNA obtained from direct sputum vs. those of sequencing from Mtb DNA obtained from culture isolates, showed that the amount of reads for Mtb was much higher from the latter samples (Table 2). The higher number of reads translated into a higher number of resistance genes identified. 
Table 1. Microbial identification through sequencing DNA analysis. LAM: Latin American and Mediterranean, WIMP: What's In My Pot.

\begin{tabular}{cccc}
\hline \multirow{2}{*}{ Samples } & Source & \multicolumn{2}{c}{ WIMP Species Identification } \\
\cline { 3 - 4 } & & Bacteria & Eukaryota \\
\cline { 3 - 4 } & & Mycobacterium tuberculosis & Homo sapiens \\
\hline HN878 & Genomic DNA (bei-resources) & 28,090 & 0 \\
LAM & Clinical isolate & 8066 & 8 \\
Beijing & Clinical isolate & 1772 & 2 \\
410 & Clinical isolate & 6736 & 376 \\
6548 & Clinical isolate & 9664 & 39 \\
1766 & Sputum & 53 & 420,062 \\
2836 & Sputum & 16 & 56,450 \\
\hline
\end{tabular}

Data shown as cumulative reads.

Table 2. Mutations observed in drug resistance (DR) related genes through MinION and previous whole genome analysis (WGA) sequencing analysis.

\begin{tabular}{|c|c|c|c|c|c|}
\hline \multirow{2}{*}{ Samples } & \multirow[b]{2}{*}{ Source } & \multicolumn{3}{|c|}{ DR Related Genes } & \multirow{2}{*}{$\begin{array}{l}\text { Phenotypic } \\
\text { Resistance } \\
\text { Validation }\end{array}$} \\
\hline & & Minion & WGA $^{a}$ & $\begin{array}{c}\text { Detected by } \\
\text { both Systems }\end{array}$ & \\
\hline HN878 & Genomic DNA (bei-resources) & 33 & 0 & - & - \\
\hline LAM & Clinical isolate & 20 & N/A & N/A & N/A \\
\hline Beijing & Clinical isolate & 32 & N/A & N/A & N/A \\
\hline 410 & Clinical isolate & 34 & 5 & 4 & $3 / 4^{b}$ \\
\hline 6548 & Clinical isolate & 29 & 6 & 6 & $6 / 6$ \\
\hline 1766 & Sputum & 5 & 3 & 1 & $1 / 3$ \\
\hline 2836 & Sputum & 3 & N/A & N/A & N/A \\
\hline
\end{tabular}

N/A: not available. ${ }^{a}$ Ref $24{ }^{a}$ One of the concordant hits among the sequencing experiments corresponded to the $e m b B$ gene, which has poor evidence of a correlation with phenotypic drug resistance; in accordance, the strain was susceptible to ethambutol in vitro. Full description of the results is available in Appendix A, Appendix B, Appendix C, Appendix D, Appendix E, Appendix F, Appendix G.

As pointed out previously, a limitation imposed by sequencing Mtb DNA from sputum samples was the high proportion of human DNA. Despite this relatively low availability of Mtb DNA for sequencing, it proved to be sufficient for obtaining a read coverage that allowed the identification of drug resistance mutations (Table 2).

We then aimed to compare the results obtained for a subset of samples for which whole genome analysis (WGA) data were available. Analyzing the MinION reads in real time with the ARMA pipeline identified a larger number of mutations in genes related to drug resistance, which in some cases included all, or the majority, of those identified by WGS analysis (Table 2). Most of the identified genes had no or poor evidence of their involvement in clinically relevant drug resistance in TB (Appendix A, Appendix B, Appendix C, Appendix D, Appendix E, Appendix F, Appendix G). For those genes with moderate or high-level evidence for drug resistance prediction, some were not supported by the drug susceptibility testing results (Table 2) or were redundant hits. For example, for isolate 6548, isoniazid (INH) resistance was attributed to $k a t G$ (also found in a previous WGA) but also to $i n h A$ and mutations in the 16S rRNA gene were listed for amikacin, streptomycin and kanamycin resistance independently.

\section{Discussion}

In this study we have evaluated the genomic identification and drug mutation gene profiling of Mtb isolates utilizing the MinION portable sequencer. Our findings endorse the need of further research regarding the practical use of MinION for the detection and characterization of Mtb in clinical isolates and in sputum samples. Our sample set consisted of Mtb genomic DNA obtained by different 
extraction methods. Recently, low-cost DNA extraction methods for Mtb WGS directly from patient samples have been reported [10], allowing the bypass of laboratory equipment requirements for genomic DNA obtainment.

The portable WGS-based detection system utilized here proved to be fast, relatively inexpensive, with rapid and simple library preparation, and automated real-time analysis tools [10]. The most innovative aspect of this sequencing system is its portability. Its small size and use of a USB port are ideal as they reduce the infrastructure required for WGS sequencing, such as a climate-controlled building, instead requiring only a laptop computer for the system to be operational [9,12].

The MinION has several advantages that make it uniquely suited for TB surveillance (Supplement Table S1). Amongst its features, the MinION provides long-read sequencing data, which are ideal for the detection of antimicrobial resistance genes [13], and some authors suggest that this can be achieved even without the need of a high amount of reads [14]. The real time monitoring allows the analysis of metagenomes from complex samples, which could save the 14 days of culture required for drug susceptibility testing in TB. In our set of DNA samples obtained directly from sputum, the presence of host DNA was far more abundant than Mtb DNA, but bacterial DNA could be discriminated and drug resistance related genes were detected, albeit at low sequencing depth. Although identification at the MTBC level provided by Xpert and other fast methods is usually enough for the diagnosis of TB, direct species assignment from sputum samples is an advantage to highlight. Another big challenge in the clinical setting is the bioinformatics analysis, as most clinical labs do not have trained personnel. Real time antimicrobial resistance profiling is indeed, a crucial advantage to highlight. The steps from raw data acquisition to analysis completion are fairly simple and easy to follow in their user-friendly EPI2ME platform [15]. Furthermore, the analysis can be performed in real time even from the moment data acquisition begins, potentially minimizing the results waiting time even more.

The number of mutations in drug resistance related genes overly surpassed those detected in previous WGA. This may have several explanations. First, a high error rate has been acknowledged as a limitation of Nanopore technology [16], thus, some of these could correspond to sequencing errors, in spite of the overall accuracy of around $90 \%$, according to the automated results. The initial high error rates reported for the MinION [17], have improved over the past few years [18], currently over 95\% raw read accuracy and $99.9 \%$ consensus read accuracy is achievable. Incorporation of complementary short read sequences [18], and the use of short DNA target sequences, circularized and then amplified via rolling-circle amplification to produce high fidelity accurate repeats [19], are new proposed ways to reduce the error rate. Additionally, recent statistical methods have been reported to aid in the accurate detection of true mutations [20] Long read sequencing has a superior advantage over short read WGS approach, especially in homopolymeric regions where indel is commonly used by bacteria as a drug resistance strategy [21]. Therefore, although the higher number of drug resistance (DR) related genes found in this study using MinION may be due in part a high error rate, it is also reasonable to think that more genes were detected by the long read sequencing compared to the traditional short read WGS. Further investigation is needed to clarify this. Additionally, it would be interesting to follow up on a newer version (R10) of Nanopore's Flowcell compared to the version used in this study (R9), as improved accuracy with longer barrel and dual reader head in the sequencing pores shall provide better accuracy especially in homopolymer regions. Alternatively, the higher number of detected DR genes by the MinION could correspond to false positive hits detected by the automated ARMA pipeline. The WHO endorses the use of next-generation sequencing analysis for drug-resistance profiling, only for a limited number of genes $(r p o B, k a t G$ and $i n h A$ for first line drugs and $g y r A, g y r B, r s s$ and eis promoters for second line drugs) and for specific point mutations within them [16]. However, the reference database used by the ARMA pipeline includes genes that lack empirical support for their clinical relevance in TB $[16,22]$. Almost half of the "hits" corresponded to this category (see Appendix A, Appendix B, Appendix C, Appendix D, Appendix E, Appendix F, Appendix G), indicating that the reference database needs further curation. In addition, some mutations in known resistance conferring genes could correspond to polymorphisms with no functional impact depending on the mutated codon (this is not disclosed in the 
automated analysis) [16], which could explain the detection of resistance related genes in susceptible isolates. The same could be said for genes like $m \operatorname{tr} A$ or AAC(2')-IC, which were detected in 5 out of 7 isolates irrespective of their resistance profile and could correspond to polymorphisms.

Nevertheless, the sensitivity of the MinION sequencing for the detection of drug resistance mutations was good. Isolates 410 and 6548 belong to the extensively studied MDR M strain [23,24] which accumulated resistance to several drugs. The ARMA pipeline detected three of the four drug resistance conferring mutations and an additional mutation in isolate 410 , and all six resistance mutations of isolate 6548. Interestingly, the gidB mutation, which confers resistance to streptomycin, is not the most frequent among clinical isolates but is characteristic of this cluster and was acquired four decades ago when the expansion of this cluster began [24]. In addition, a rifampicin resistance conferring mutation was found in the metagenome of the sputum sample 1766, which belongs to the Ra cluster, another conspicuous MDR strain of Argentina [25]. These phenotypically confirmed drug-resistance conferring mutations were identified with two to 17 reads depending on the gene, with similar accuracy values. This indicates that although it is usually regarded a critical variable in the analysis of next-generation sequencing data, sequencing depth was not the main constraint in our work. Prompt and accurate information on $\mathrm{Mtb}$ strains would have implications for management to minimize transmission of drug-resistant TB and start the most appropriate TB control and anti-TB therapy. Various phylogenetic lineages of the Mtb complex are distributed differently around the world [2]. In Latin America, both drug susceptible and drug resistant TB are mainly related to the Euro-American Lineage [26-29] and the Beijing strain has a minor impact, in contrast to what is reported in other regions. Drug resistance databases mostly rely on the genome H37Rv strain. It is interesting to challenge this sequencing system with samples sets with diverse genetic backgrounds like ours to assess its impact in the performance.

Overall, our findings indicate that the improvements in the future should focus on: (1) recovering higher number of reads corresponding to Mtb from sputa; (2) lowering MinION sequencing error rates; (3) improving the drug-resistance conferring mutation detection algorithms for automated analysis and (4) curating the reference database to include only those hits that have a strong correlation with Mtb drug resistance phenotype.

Although our data relies on a short number of DNA samples, our findings suggest that this portable DNA sequencing system could be effective in reducing time and providing information on Mtb genotype and drug-susceptibility from direct sputum samples. As larger studies-evaluating parameters such as the minimal number of reads for a complete reliable drug susceptibility profiling, optimization in software and database accuracy for the prediction of new drug resistance genes, and reduction in false positive drug detection-are conducted, this system could potentially revolutionize current TB testing procedures, especially in genomic surveillance for MDR-TB in the clinical setting.

\section{Materials and Methods}

\subsection{Mtb Genomic DNA}

Mtb genomic DNA, strain HN878 was acquired through bei resources (NR-14867). Genomic bacterial DNA extracted from four laboratory cultured Mtb isolates from sputum samples was also utilized (Table 1). These correspond to a strain belonging to the Beijing lineage, a strain belonging to the Latin American and Mediterranean (LAM) lineage [27], and two closely related strains belonging to the Haarlem lineage, the so called M strain (isolate 6548), and an M strain variant (isolate 410). Genomic DNA was also extracted directly from 2 sputum samples from pulmonary TB patients with positive bacilloscopy scored through correspondent acid-fast bacteria (AFB) smears. These latter samples included a strain susceptible to the first line drugs INH, RIF, STR, EMB (sample 2836) and an Ra strain (sample 1766) which along with the M strain constitute the most prevalent MDR clusters in Argentina [30]. MPure ${ }^{\mathrm{TM}}$ DNA Extraction Kit (MP Biomedical), as well as inactivation and lysis by sonication protocol [27] were used for bacterial DNA extraction, except for DNA from strain HN878, which used a delipidation method, followed by lysozyme, RNase, SDS and proteinase digestion [31]. 


\subsection{Whole Genome Sequencing (WGS) Data}

Whole genome sequencing (WGS) with Illumina was available for isolates 6548 and 410 [23] and for representative isolates of the Ra cluster for comparison with sputum sample 1766 [22]. WGS data from Mtb was obtained by eliminating human DNA sequences utilizing "Read Until" approach (OMICtools) for target sequencing [32]. Mtb identification was performed once the metagenome was obtained.

\subsection{MinION DNA Sequencing and Resistance Gene Identification}

DNA sample libraries were constructed using Rapid Sequencing Kit (ONT, Littlemore, UK), and sequencing was conducted on MinION-compatible R9.4 flow cells (ONT, UK). Primary data acquisition was done using MinKNOW, the operating software that drives nanopore sequencing devices. Raw data were processed for basecalling via Albacore. Data were then further processed using the cloud-based data analysis platform EPI2ME [15]. Microbial species identification was done using the What's In My Pot (WIMP) analysis workflow [11], and detection of mutations conferring antibiotic drug resistance was done through the real time antimicrobial resistance mapping application (ARMA) [33].

\section{Conclusions}

In this study we have evaluated the genomic identification and drug mutation gene profiling of Mtb isolates utilizing the MinION portable sequencer The approach was highly sensitive, being able to detect almost all described drug resistance conferring mutations based on previous whole genome sequencing analysis. Our findings are supportive of the practical use of this system as a suitable method for the detection of antimicrobial resistance genes, and effective in providing Mtb genomic information. Future improvements in the error rate through statistical analysis, drug resistance prediction algorithms and reference databases would make this a platform suited for the clinical setting. The small size, relatively inexpensive cost of the device, as well as its rapid and simple library preparation protocol and analysis, make it an attractive option for settings with limited laboratory infrastructure.

Supplementary Materials: The following are available online at http://www.mdpi.com/2079-6382/9/9/548/s1, Table S1: Comparison of MinION based TB surveillance and current probe based or culture-based methods.

Author Contributions: Conceptualization, J.C., N.Y. and B.-Y.H.; methodology, J.C., N.Y. and B.-Y.H.; data analysis J.C., N.Y. and B.-Y.H.; manuscript preparation J.C., N.Y. and B.-Y.H. All authors have read and agreed to the published version of the manuscript.

Funding: This research received no external funding.

Acknowledgments: Special thanks to María Elvira Balcells (Medicina. Pontificia Universidad Católica de Chile), Viviana Ritacco and Johana Monteserin (CONICET, ANLIS “DR. C. G. Malbrán”) for sharing their samples and expertise. Many thanks to Jose Barragan (TTUHSC-LEMM) and Miki Wang (PLFSOM, TTUHSC) for their technical assistance, and to Melissa Huddleston (PLFSOM, TTUHSC) for her help in editing the manuscript.

Conflicts of Interest: The authors declare no conflict of interest. 


\section{Appendix A}

\begin{tabular}{|c|c|c|c|c|c|}
\hline & $\begin{array}{l}\text { Specimen } \\
\text { DR Status } \\
\text { Strain }\end{array}$ & $\begin{array}{c}\text { ic DNA } \\
\text { MDR } \\
\text { HN878 }\end{array}$ & & & \\
\hline \multicolumn{6}{|c|}{ MinION } \\
\hline & DR GENE & Drug & Comments $^{1}$ & Reads & Accuracy \\
\hline \multicolumn{6}{|c|}{ Drug resistance related genes } \\
\hline 1 & rpoB & RIF & Strong evidence for rifampicin resistance & 138 & 90.8 \\
\hline 2 & gyrA & FLQ & Strong evidence. Most commonly associated with FLQ resistance & 110 & 90.8 \\
\hline 3 & ethA & ETH & Low level evidence & 107 & 91.3 \\
\hline 4 & $\operatorname{gidB}$ & STR & Low level evidence for strains other than the $\mathrm{M}$ strain & 88 & 90.9 \\
\hline 5 & katG & INH & Strong evidence for high level resistance to INH & 84 & 90.5 \\
\hline 6 & $\operatorname{inh} A$ & INH & Strong evidence for low level resistance to INH & 74 & 91.3 \\
\hline 7 & pncA & PZA & Strong evidence for pyrazinamide resistance & 73 & 91.5 \\
\hline 8 & $e m b A$ & EMB & Prom -12 related to ethambutol $R$ but not enough evidence & 73 & 90.7 \\
\hline 9 & tlyA & Capreo & Related to capreomycin resistance & 69 & 90.8 \\
\hline 10 & $e m b B$ & EMB & Most frequently found mutation, but not enough evidence for clinical use & 68 & 91.5 \\
\hline 11 & kas $A$ & INH & Not a frequent mutation conferring gene, low level of evidence & 63 & 90.7 \\
\hline 12 & $r p s L$ & STR & Strong evidence for STR resistance & 58 & 91 \\
\hline 13 & 16S rRNA & STR & Frequently found for STR R (in nt different from 2nd line injectables) & 32 & 90.9 \\
\hline 14 & $16 \mathrm{~S}$ rRNA & KAN & Strong evidence for resistance to all 32 nd line injectables & 27 & 90.4 \\
\hline 15 & 16S rRNA & AMK & Strong evidence for resistance to all 32 nd line injectables & 20 & 90.3 \\
\hline 16 & gyrB & FLQ & Strong evidence, related to FLQ resistance & 77 & 90.9 \\
\hline 17 & $e m b C$ & $\mathrm{EMB}$ & Related to ethambutol resistance but not enough evidence for clinical use & 60 & 90.5 \\
\hline
\end{tabular}




\begin{tabular}{|c|c|c|c|c|c|}
\hline \multicolumn{6}{|c|}{ No evidence for involvement in clinically relevant drug resistance } \\
\hline 18 & efp $A$ & efflux pump & No evidence of involvement in drug resistant phenotype & 93 & 91.3 \\
\hline 19 & $e m b R$ & EMB & No evidence of involvement in drug resistant phenotype & 82 & 91.1 \\
\hline 20 & $A A C\left(2^{\prime}\right)-I c$ & EMB & No evidence of involvement in drug resistant phenotype & 75 & 92 \\
\hline 21 & $n d h$ & INH & No evidence of involvement in drug resistant phenotype & 74 & 90.9 \\
\hline 22 & iniA & EMB & Induced by drugs but role in resistance unclear & 74 & 91.9 \\
\hline 23 & $\operatorname{Erm}(37)$ & - & No evidence of involvement in drug resistant phenotype & 70 & 90.9 \\
\hline 24 & $m \operatorname{tr} A$ & - & No evidence of involvement in drug resistant phenotype & 69 & 91.3 \\
\hline 25 & $m f p A$ & - & No evidence of involvement in drug resistant phenotype & 67 & 91.3 \\
\hline 26 & tsnr & - & No evidence of involvement in drug resistant phenotype & 66 & 89.8 \\
\hline 27 & $d r r A$ & - & No evidence of involvement in first or second line drug resistance & 63 & 92.2 \\
\hline 28 & iniC & EMB & Induced by drugs but role in resistance unclear & 57 & 91 \\
\hline 29 & $\operatorname{drrC}$ & - & No evidence of involvement in first or second line drug resistance & 45 & 91.9 \\
\hline 30 & murA & - & No evidence of involvement in drug resistant phenotype & 44 & 90.3 \\
\hline 31 & $d r r B$ & - & No evidence of involvement in first or second line drug resistance & 36 & 92.5 \\
\hline 32 & arabinosyltransferase & - & No evidence of involvement in drug resistant phenotype & 31 & 92.6 \\
\hline 33 & 16S rRNA & Viomycin & Not a 1st or 2nd line drug for TB & 27 & 90 \\
\hline
\end{tabular}

1 Relevance in DR was checked in mycobrowser.epfl.ch, in Boritsch and Brosch 2016 Microbiology spectrum (10.1128/microbiolspec.TBTB2-0020-2016) and https://apps.who.int/iris/handle/ 10665/274443; AMK: amikacin, Capreo: capreomycin, EMB: ethambutol, ETH: ethinamide, FLQ: fluoroquinolones, INH: isoniazid, KAN: kanamycin, PZA: pyrazinamide, RIF: rifampicin, STR: streptomycin, TB: tuberculosis. 


\section{Appendix B}

\begin{tabular}{|c|c|c|c|c|c|}
\hline \multicolumn{6}{|c|}{ Specimen: Culture isolate } \\
\hline & $\begin{array}{l}\text { DR Status } \\
\text { Strain }\end{array}$ & Susceptible & $\begin{array}{l}\text { Resistance Profile: PanS } \\
\text { LAM Family Strain }\end{array}$ & & \\
\hline \multicolumn{6}{|c|}{ MinION } \\
\hline & DR GENE & Drug & Comments $^{1}$ & Reads & Accuracy \\
\hline \multicolumn{6}{|c|}{ Drug resistence related genes } \\
\hline 1 & $e m b A$ & EMB & prom -12 related to ethambutol $\mathrm{R}$ but not enough evidence & 5 & 86.3 \\
\hline 2 & $e m b C$ & EMB & Related to ethambutol resistance but not enough evidence for clinical use & 3 & 89 \\
\hline 3 & tlyA & Capreo & Related to capreomycin resistance & 2 & 82.7 \\
\hline 4 & $e m b B$ & EMB & Most frequently found mutation, but not enough evidence for clinical use & 2 & 88.2 \\
\hline 5 & $r p o B$ & RIF & strong evidence for rifampicin resistance & 2 & 89.3 \\
\hline 6 & $g y r B$ & FLQ & Strong evidence, related to FLQ resistance & 2 & 91.3 \\
\hline 7 & ethA & ETH & Low level evidence & 2 & 95.4 \\
\hline 8 & katG & INH & Strong evidence for high level resistance to INH & 1 & 91.9 \\
\hline 9 & $r p s L$ & STR & Strong evidence for STR resistance & 1 & 90.3 \\
\hline 10 & kas $A$ & INH & Not a frequent mutation conferring gene, low level of evidence & 1 & 91.9 \\
\hline \multicolumn{6}{|c|}{ No evidence for involvement in clinically relevant drug resistance } \\
\hline 11 & $m \operatorname{tr} A$ & - & No evidence of involvement in drug resistant phenotype & 3 & 91 \\
\hline 12 & murA & - & No evidence of involvement in drug resistant phenotype & 2 & 87.8 \\
\hline 13 & AAC $\left(2^{\prime}\right)-\mathrm{Ic}$ & - & No evidence of involvement in drug resistant phenotype & 2 & 87.8 \\
\hline 14 & $n d h$ & INH & No evidence of involvement in drug resistant phenotype & 2 & 83.6 \\
\hline 15 & iniA & EMB & Induced by drugs but role in resistance unclear & 2 & 85.7 \\
\hline 16 & iniC & EMB & Induced by drugs but role in resistance unclear & 2 & 87.4 \\
\hline 17 & $e m b B$ & RIF & Not related to RIF R & 2 & 91.8 \\
\hline 18 & tsnr & - & No evidence of involvement in drug resistant phenotype & 1 & 85 \\
\hline 19 & $e m b R$ & EMB & No evidence of involvement in drug resistant phenotype & 1 & 85.8 \\
\hline 20 & arabinosyltransferase & - & No evidence of involvement in drug resistant phenotype & 1 & 89.4 \\
\hline
\end{tabular}

1 Relevance in DR was checked in mycobrowser.epfl.ch, in Boritsch and Brosch 2016 Microbiology spectrum (10.1128/microbiolspec.TBTB2-0020-2016) and https://apps.who.int/iris/handle/ 10665/274443; AMK: amikacin, Capreo: capreomycin, EMB: ethambutol, ETH: ethinamide, FLQ: fluoroquinolones, H/INH: isoniazid, KAN: kanamycin, Z/PZA: pyrazinamide, R/RIF: rifampicin, S/STR: streptomycin. 


\section{Appendix C}

\begin{tabular}{|c|c|c|c|c|c|}
\hline & $\begin{array}{l}\text { Specimen: Cu } \\
\text { DR Status: } \\
\text { Strain: }\end{array}$ & $\begin{array}{l}\text { isolate } \\
\text { Susceptible }\end{array}$ & Beijing family strain & & \\
\hline \multicolumn{6}{|c|}{ MinION } \\
\hline & DR GENE & Drug & Comments ${ }^{1}$ & Reads & Accuracy \\
\hline \multicolumn{6}{|c|}{ Drug resistance related genes } \\
\hline 1 & $e m b A$ & EMB & prom -12 related to ethambutol $\mathrm{R}$ but not enought evidence & 23 & 87.8 \\
\hline 2 & $r p o B$ & RIF & strong evidence for rifampicin resistance & 20 & 88.4 \\
\hline 3 & $e m b B$ & EMB & Most frequently found mutation, but not enough evidence for clinical use & 13 & 87 \\
\hline 4 & kas $A$ & INH & Not a frequent mutation conferring gene, low level of evidence & 11 & 90.8 \\
\hline 5 & gyrB & FLQ & Strong evidence, related to FLQ resistance & 9 & 88.4 \\
\hline 6 & gyrA & FLQ & Strong evidence. Most commonly associated with FLQ resistance & 8 & 88.6 \\
\hline 7 & katG & INH & Strong evidence for high level resistance to INH & 7 & 86.1 \\
\hline 8 & ethA & ETH & Low level evidence & 7 & 92 \\
\hline 9 & $e m b C$ & EMB & Related to ethambutol resistance but not enough evidence for clinical use & 6 & 90.5 \\
\hline 10 & tlyA & Capreo & Related to capreomycin resistance & 6 & 87.5 \\
\hline 11 & inh $A$ & INH & Strong evidence for low level resistance to INH & 6 & 89.6 \\
\hline 12 & $\operatorname{gidB}$ & STR & Low level evidence for strains other than the M strain & 3 & 91.4 \\
\hline 13 & pncA & PZA & strong evidence for pyrazinamide resistance & 1 & 97.5 \\
\hline 14 & $r p s L$ & STR & Strong evidence for STR resistance & 1 & 91.6 \\
\hline 15 & $16 \mathrm{~S}$ rRNA & AMK & Strong evidence for resistance to all 32 nd line injectables & 1 & 87.3 \\
\hline \multicolumn{6}{|c|}{ No evidence for involvement in clinically relevant drug resistance } \\
\hline 16 & murA & - & No evidence of involvement in drug resistant phenotype & 10 & 86.4 \\
\hline 17 & efp $A$ & efflux pump & No evidence of involvement in drug resistant phenotype & 7 & 90.6 \\
\hline 18 & drra & - & No evidence of involvement in first or second line drug resistance & 7 & 86.5 \\
\hline 19 & $n d h$ & INH & No evidence of involvement in drug resistant phenotype & 7 & 92.6 \\
\hline 20 & $e m b R$ & EMB & No evidence of involvement in drug resistant phenotype & 6 & 92 \\
\hline 21 & $\operatorname{Erm}(37)$ & - & No evidence of involvement in drug resistant phenotype & 5 & 90.3 \\
\hline 22 & $d r r C$ & - & No evidence of involvement in first or second line drug resistance & 5 & 89.9 \\
\hline 23 & $\operatorname{drr} B$ & - & No evidence of involvement in first or second line drug resistance & 5 & 86.5 \\
\hline 24 & $m f p A$ & - & No evidence of involvement in drug resistant phenotype & 4 & 88.7 \\
\hline 25 & mtrA & - & No evidence of involvement in drug resistant phenotype & 4 & 89 \\
\hline 26 & iniA & EMB & Induced by drugs but role in resistance unclear & 4 & 93.7 \\
\hline 27 & iniC & EMB & Induced by drugs but role in resistance unclear & 4 & 89.4 \\
\hline 28 & tsnr & - & No evidence of involvement in drug resistant phenotype & 3 & 86.4 \\
\hline 29 & $A A C\left(2^{\prime}\right)-I c$ & - & No evidence of involvement in drug resistant phenotype & 2 & 87.2 \\
\hline 30 & arabinosyltransferase & - & No evidence of involvement in drug resistant phenotype & 2 & 91.1 \\
\hline 31 & $E F-T u$ & Elfamycin & Not a 1st or 2nd line drug for $\mathrm{TB}$ & 1 & 74.3 \\
\hline 32 & $16 \mathrm{~S}$ rRNA & Viomycin & Not a 1st or 2nd line drug for $\mathrm{TB}$ & 1 & 84.8 \\
\hline
\end{tabular}

1 Relevance in DR was checked in mycobrowser.epfl.ch, in Boritsch and Brosch 2016 Microbiology spectrum (10.1128/microbiolspec.TBTB2-0020-2016) and https://apps.who.int/iris/handle/ 10665/274443; AMK: amikacin, Capreo: capreomycin, EMB: ethambutol, ETH: ethinamide, FLQ: fluoroquinolones, INH: isoniazid, KAN: kanamycin, PZA: pyrazinamide, RIF: rifampicin, STR: streptomycin. 


\section{Appendix D}

\begin{tabular}{|c|c|c|c|c|c|c|c|c|c|}
\hline & $\begin{array}{l}\text { Sample: } 410 \\
\text { DR Status: } \\
\text { Strain: }\end{array}$ & MDR & $\begin{array}{l}\text { Specimen: Isolate } \\
\text { Resistance profie: INH, RIF, STR, PZA } \\
\text { M strain-variant }\end{array}$ & & & & & & \\
\hline & & & MinION & \multicolumn{5}{|c|}{ WGA $^{2}$} & \\
\hline & DR GENE & Drug & Comments $^{1}$ & READS & Accuracy & Mutation & MinION vs. WGA & DR Phenotype & MinION vs. Phenotype \\
\hline \multicolumn{10}{|c|}{ Drug resistence related genes } \\
\hline 1 & kas $A$ & $\mathrm{INH}$ & Not a frequent mutation conferring gene, low level of evidence & 20 & 89.2 & $\mathrm{wt}$ & discordant & $\mathrm{R}$ & - \\
\hline 2 & gyrA & FLQ & Strong evidence. Most commonly associated with FLQ resistance & 16 & 91.6 & wt & discordant & $\mathrm{s}$ & discordant \\
\hline 3 & rрoв & RIF & strong evidence for rifampicin resistance & 16 & 89.3 & $\mathrm{H} 526 \mathrm{~L}^{4}$ & concordant & $\mathbf{R}$ & concordant \\
\hline 4 & pnca & PZA & strong evidence for pyrazinamide resistance & 14 & 91 & Y103D & concordant & $\mathbf{R}$ & concordant \\
\hline 5 & $\operatorname{gidB}$ & STR & V100fs is a unique mutation found in M strain lineage ${ }^{3}$ & 14 & 89.7 & V100fs & concordant & $\mathbf{R}$ & concordant \\
\hline 6 & thy & Capreo & Related to capreomycin resistance & 13 & 91 & wt & discordant & $\mathrm{s}$ & discordant \\
\hline 7 & $r p s L$ & STR & Strong evidence for STR resistance & 12 & 92.6 & wt & discordant & - & - \\
\hline 8 & $\operatorname{inh} A$ & INH & Strong evidence for low level resistance to INH & 12 & 91.6 & wt & discordant & - & - \\
\hline 9 & gyrB & FLQ & Strong evidence, related to FLQ resistance & 10 & 89.9 & wt & discordant & $\mathrm{s}$ & discordant \\
\hline 10 & ethA & ETH & $\begin{array}{l}\text { Low level evidence } \\
\text { L }\end{array}$ & 10 & 88.1 & $\mathrm{wt}$ & discordant & $\mathrm{s}$ & discordant \\
\hline 11 & $e m b B$ & EMB & Most frequently found mutation, but not enough evidence for clinical use & 7 & 88.7 & $\mathrm{wt}$ & discordant & $\mathrm{s}$ & discordant \\
\hline 12 & $e m b C$ & ЕМв & Related to ethambutol resistance but not enough evidence for clinical use & 6 & 89.4 & V981L & concordant & $\mathrm{s}$ & no strong evidence of \\
\hline 13 & embA & EMB & prom -12 related to ethambutol R but not enought evidence & 5 & 89 & wt & discordant & $\mathrm{s}$ & discordant \\
\hline 14 & $16 \mathrm{~S}$ rRNA & STR & Frequently found for STR $\mathrm{R}$ (in $\mathrm{nt}$ different from 2nd line injectables) & 4 & 89.4 & wt & discordant & $\mathrm{s}$ & discordant \\
\hline 15 & $16 \mathrm{~S}$ rRNA & AMK & Strong evidence for resistance to all 32 nd line injectables & 3 & 91.1 & wt & discordant & $\mathrm{s}$ & discordant \\
\hline 16 & $16 \mathrm{~S}$ rRNA & KAN & Strong evidence for resistance to all 3 2nd line injectables & 3 & 88.3 & wt & discordant & $\mathrm{s}$ & discordant \\
\hline 17 & katG & INH & Strong evidence for high level resistance to INH & undetected & d - & S315T & discordant & $\mathbf{R}$ & discordant (gene undetected) \\
\hline \multicolumn{10}{|c|}{ No evidence for involvement in clinically relevant drug resistance } \\
\hline 18 & $m t r A$ & & Not related to DR & 19 & 87.9 & $\mathrm{wt}$ & discordant & - & - \\
\hline 19 & iniA & EMB & Induced by drugs but role in resistance unclear & 16 & 88.3 & wt & discordant & - & - \\
\hline 20 & tsur & - & Not related to DR & 16 & 88.5 & $\mathrm{wt}$ & discordant & - & - \\
\hline 21 & $\operatorname{Erm}(37)$ & - & Not related to DR & 15 & 87.9 & wt & discordant & - & - \\
\hline 22 & $m f p A$ & - & Not related to DR & 15 & 90.9 & $\mathrm{wt}$ & discordant & - & - \\
\hline 23 & $e m b R$ & EMB & No evidence of involvement in drug resistant phenotype & 13 & 89.6 & $\mathrm{wt}$ & discordant & - & - \\
\hline 24 & efpA & efflux pump & No evidence of involvement in drug resistant phenotype & 11 & 90.5 & wt & discordant & - & - \\
\hline 25 & $\mathrm{AAC}\left(2^{\prime}\right)-\mathrm{Ic}$ & & Not related to DR & 10 & 89.5 & wt & discordant & - & - \\
\hline 26 & drra & - & No evidence of involvement in first or second line drug resistance & 10 & 91.4 & wt & discordant & - & - \\
\hline 27 & $n d h$ & $\mathrm{INH}$ & No evidence of involvement in drug resistant phenotype & 9 & 89.7 & $\mathrm{wt}$ & discordant & - & - \\
\hline 28 & mur $A$ & - & No evidence of involvement in drug resistant phenotype & 8 & 87.5 & $\mathrm{wt}$ & discordant & - & - \\
\hline 29 & $d r r C$ & - & No evidence of involvement in first or second line drug resistance & 5 & 93 & $\mathrm{wt}$ & discordant & - & - \\
\hline 30 & inic & EMB & Induce by drugs but role in resistance unclear & 4 & 86.5 & wt & discordant & - & - \\
\hline 31 & $16 \mathrm{~S}$ rRNA & viomycin & Not a 1st or 2nd line drug for TB & 4 & 92.1 & wt & discordant & - & - \\
\hline 32 & $d r r B$ & - & No evidence of involvement in first or second line drug resistance & 3 & 92.2 & $\mathrm{wt}$ & discordant & - & - \\
\hline 33 & $e m b B$ & RIF & Not related to RIF R & 3 & 91.1 & $\mathrm{wt}$ & discordant & - & - \\
\hline 34 & RbpA & & Not related to DR in Mtb & 2 & 79.5 & wt & discordant & - & - \\
\hline
\end{tabular}

1 Relevance in DR was checked in mycobrowser.epfl.ch, in Boritsch and Brosch, 2016, Microbiology spectrum (10.1128/microbiolspec.TBTB2-0020-2016) and https://apps.who.int/iris/ handle/10665/274443; 2 Bigi et al. 2017 Tuberculosis (Edinb.) 103 28-36; 3 Eldhom et al. 2015 Nature Comm; 4 E. coli annotation; AMK: amikacin, Capreo: capreomycin, EMB: ethambutol,
ETH: ethinamide, FLQ: fluoroquinolones, INH: isoniazid, KAN: kanamycin, PZA: pyrazinamide, RIF: rifampicin, STR: streptomycin. R: resistant, S: susceptible; Bold letters correspond to drug resistance related mutations previously found in 410 strain. 


\section{Appendix E}

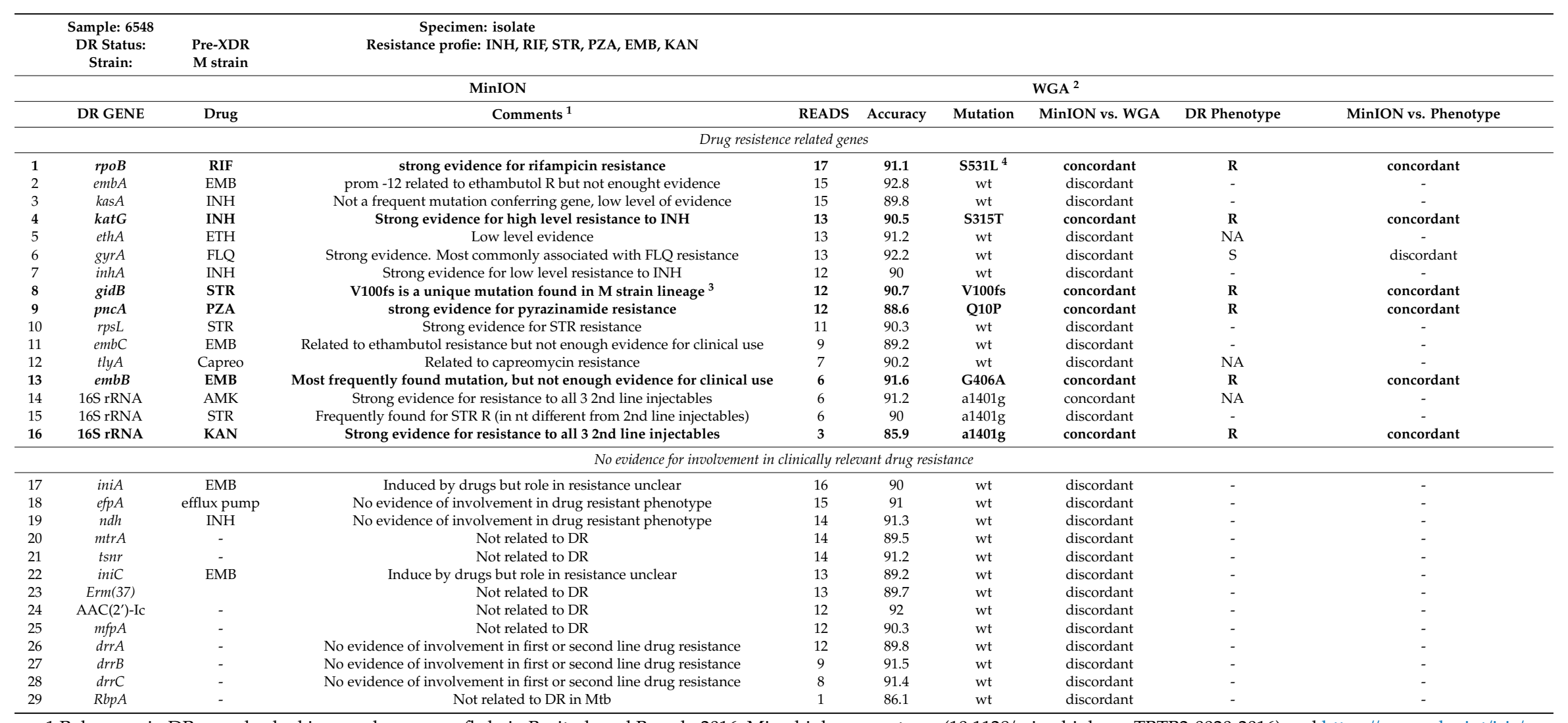

1 Relevance in DR was checked in mycobrowser.epfl.ch, in Boritsch and Brosch, 2016, Microbiology spectrum (10.1128/microbiolspec.TBTB2-0020-2016) and https://apps.who.int/iris/ handle/10665/274443; 2 Bigi et al. 2017 Tuberculosis (Edinb.) 103 28-36; 3 Eldhom et al. 2015 Nature Comm; 4 E. coli annotation; AMK: amikacin, Capreo: capreomycin, EMB: ethambutol, ETH: ethinamide, FLQ: fluoroquinolones, INH: isoniazid, KAN: kanamycin, PZA: pyrazinamide, RIF: rifampicin, STR: streptomycin. R: resistant, S: susceptible; Bold letters correspond to drug resistance related mutations found in $\mathrm{M}$ strain. 


\section{Appendix F}

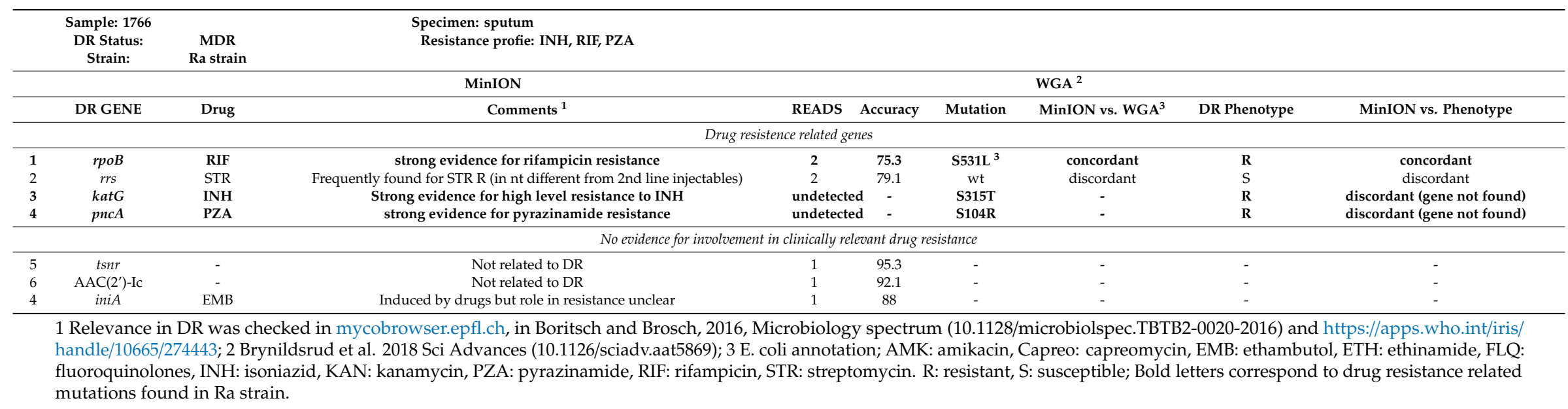

\section{Appendix G}

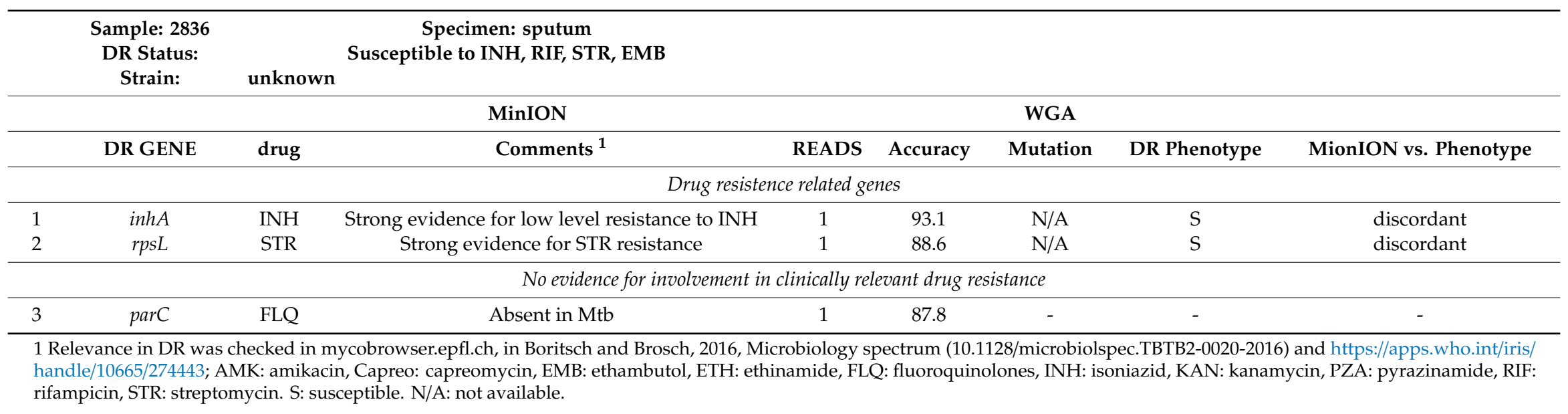




\section{References}

1. Daley, C.L. The Global Fight against Tuberculosis. Thorac. Surg. Clin. 2019, 29, 19-25. [CrossRef] [PubMed]

2. Gagneux, S. Ecology and evolution of Mycobacterium tuberculosis. Nat. Rev. Microbiol. 2018, 16, $202-213$. [CrossRef]

3. The CRyPTIC Consortium and the 100,000 Genomes Project. Prediction of Susceptibility to First-Line Tuberculosis Drugs by DNA Sequencing. N. Engl. J. Med. 2018, 379, 1403-1415. [CrossRef] [PubMed]

4. Koch, A.; Cox, H.; Mizrahi, V. Drug-resistant tuberculosis: Challenges and opportunities for diagnosis and treatment. Curr. Opin. Pharmacol. 2018, 42,7-15. [CrossRef]

5. World Health Organization. High-Priority Target Product Profiles for New Tuberculosis Diagnostics: Report of a Consensus Meeting; Contract No.: WHO/HTM/TB/2014.18; World Health Organization: Geneva, Switzerland, 2014.

6. Sanchez-Padilla, E.; Merker, M.; Beckert, P.; Jochims, F.; Dlamini, T.; Kahn, P.; Bonnet, M.; Niemann, S. Detection of drug-resistant tuberculosis by Xpert MTB/RIF in Swaziland. N. Engl. J. Med. 2015, 372, 1181-1182. [CrossRef] [PubMed]

7. Ocheretina, O.; Escuyer, V.E.; Mabou, M.M.; Royal-Mardi, G.; Collins, S.; Vilbrun, S.C.; Pape, J.W.; Fitzgerald, D.W. Correlation between genotypic and phenotypic testing for resistance to rifampin in Mycobacterium tuberculosis clinical isolates in Haiti: Investigation of cases with discrepant susceptibility results. PLoS ONE 2014, 9, e90569. [CrossRef]

8. Doyle, R.M.; Burgess, C.; Williams, R.; Gorton, R.; Booth, H.; Brown, J.; Bryant, J.M.; Chan, J.; Creer, D.; Holdstock, J.; et al. Direct Whole-Genome Sequencing of Sputum Accurately Identifies Drug-Resistant Mycobacterium tuberculosis Faster than MGIT Culture Sequencing. J. Clin. Microbiol. 2018, 56. [CrossRef]

9. Runtuwene, L.R.; Tuda, J.S.B.; Mongan, A.E.; Suzuki, Y. On-Site MinION Sequencing. Adv. Exp. Med. Biol. 2019, 1129, 143-150.

10. Votintseva, A.A.; Bradley, P.; Pankhurst, L.; Del Ojo Elias, C.; Loose, M.; Nilgiriwala, K.; Chatterjee, A.; Smith, E.G.; Sanderson, N.; Walker, T.M.; et al. Same-Day Diagnostic and Surveillance Data for Tuberculosis via Whole-Genome Sequencing of Direct Respiratory Samples. J. Clin. Microbiol. 2017, 55, 1285-1298. [CrossRef]

11. Juul, S.; Izquierdo, F.; Hurst, A.; Dai, X.; Wright, A.; Kulesha, E.; Pettett, R.; Turner, D.J. What's in my pot? Real-time species identification on the MinION'T . Biorxiv 2015. [CrossRef]

12. Wolkowicz, T. The utility and perspectives of NGS-based methods in BSL-3 and BSL-4 laboratory-sequencing and analysis strategies. Brief. Funct. Genomics 2018, 17, 471-476. [CrossRef] [PubMed]

13. Judge, K.; Harris, S.R.; Reuter, S.; Parkhill, J.; Peacock, S.J. Early insights into the potential of the Oxford Nanopore MinION for the detection of antimicrobial resistance genes. J. Antimicrob. Chemother. 2015, 70, 2775-2778. [CrossRef] [PubMed]

14. Bradley, P.; Gordon, N.C.; Walker, T.M.; Dunn, L.; Heys, S.; Huang, B.; Earle, S.; Pankhurst, L.J.; Anson, L.; De Cesare, M.; et al. Rapid antibiotic-resistance predictions from genome sequence data for Staphylococcus aureus and Mycobacterium tuberculosis. Nat. Commun. 2015, 6, 10063. [CrossRef]

15. Nanoporetechnologies. He EPI2ME Platform a Cloud-Based Data Analysis Service for Oxford Nanopore Technologies' Sequence Information 2020. Available online: https:/epi2me.nanoporetech.com/ (accessed on 25 April 2020).

16. World Health Organization. The Use of Next-Generation Sequencing Technologies for the Detection of Mutations Associated with Drug Resistance in Mycobacterium Tuberculosis Complex: Technical Guide; World Health Organization: Geneva, Switzerland, 2018.

17. Laver, T.; Harrison, J.; O’Neill, P.A.; Moore, K.; Farbos, A.; Paszkiewicz, K.; Studholme, D.J. Assessing the performance of the Oxford Nanopore Technologies MinION. Biomol. Detect. Quantif. 2015, 3, 1-8. [CrossRef]

18. Bainomugisa, A.; Duarte, T.; Lavu, E.; Pandey, S.; Coulter, C.; Marais, B.J.; Coin, L.M. A complete high-quality MinION nanopore assembly of an extensively drug-resistant Mycobacterium tuberculosis Beijing lineage strain identifies novel variation in repetitive PE/PPE gene regions. Microb. Genomics 2018, 4, e000188. [CrossRef] [PubMed]

19. Wilson, B.D.; Eisenstein, M.; Soh, H.T. High-Fidelity Nanopore Sequencing of Ultra-Short DNA Targets. Anal. Chem. 2019, 91, 6783-6789. [CrossRef] [PubMed]

20. Harel, N.; Meir, M.; Gophna, U.; Stern, A. Direct sequencing of RNA with MinION Nanopore: Detecting mutations based on associations. Nucleic Acids Res. 2019, 47, e148. [CrossRef] 
21. Bellerose, M.M.; Baek, S.H.; Huang, C.C.; Moss, C.E.; Koh, E.I.; Proulx, M.K.; Smith, C.M.; Baker, R.E.; Lee, J.S.; Eum, S.; et al. Common Variants in the Glycerol Kinase Gene Reduce Tuberculosis Drug Efficacy. Mbio 2019, 10. [CrossRef]

22. Boritsch, E.C.; Brosch, R. Evolution of Mycobacterium tuberculosis: New Insights into Pathogenicity and Drug Resistance. Microbiol. Spectr. 2016, 4. [CrossRef]

23. Bigi, M.M.; Lopez, B.; Blanco, F.C.; Sasiain, M.D.; De la Barrera, S.; Marti, M.A.; Sosa, E.J.; Do Porto, D.A.; Ritacco, V.; Bigi, F.; et al. Single nucleotide polymorphisms may explain the contrasting phenotypes of two variants of a multidrug-resistant Mycobacterium tuberculosis strain. Tuberculosis 2017, 103, 28-36. [CrossRef]

24. Eldholm, V.; Monteserin, J.; Rieux, A.; Lopez, B.; Sobkowiak, B.; Ritacco, V.; Balloux, F. Four decades of transmission of a multidrug-resistant Mycobacterium tuberculosis outbreak strain. Nat. Commun. 2015, 6, 1-9. [CrossRef] [PubMed]

25. Brynildsrud, O.B.; Pepperell, C.S.; Suffys, P.; Grandjean, L.; Monteserin, J.; Debech, N.; Bohlin, J.; Alfsnes, K.; Pettersson, J.O.; Kirkeleite, I.; et al. Global expansion of Mycobacterium tuberculosis lineage 4 shaped by colonial migration and local adaptation. Sci. Adv. 2018, 4, eaat5869. [CrossRef]

26. Meza, P.; Balcells, M.E.; Miranda, C.; Cifuentes, M.; Wozniak, A.; Garcia, P. Presence of Bejing genotype among Mycobacterium tuberculosis strains in two centres of the Region Metropolitana of Chile. Rev. Chil. Infectol. 2014, 31, 21-27. [CrossRef] [PubMed]

27. Balcells, M.E.; Garcia, P.; Meza, P.; Pena, C.; Cifuentes, M.; Couvin, D.; Rastogi, N. A first insight on the population structure of Mycobacterium tuberculosis complex as studied by spoligotyping and MIRU-VNTRs in Santiago, Chile. PLoS ONE 2015, 10, e0118007. [CrossRef] [PubMed]

28. Millet, J.; Streit, E.; Berchel, M.; Bomer, A.G.; Schuster, F.; Paasch, D.; Vanhomwegen, J.; Cadelis, G.; Rastogi, N. A systematic follow-up of Mycobacterium tuberculosis drug-resistance and associated genotypic lineages in the French Departments of the Americas over a seventeen-year period. Biomed Res. Int. 2014, 2014, 689852. [CrossRef] [PubMed]

29. Ritacco, V.; Iglesias, M.-J.; Ferrazoli, L.; Monteserin, J.; Dalla Costa, E.R.; Cebollada, A.; Morcillo, N.; Robledo, J.; de Waard, J.H.; Araya, P.; et al. Conspicuous multidrug-resistant Mycobacterium tuberculosis cluster strains do not trespass country borders in Latin America and Spain. Infect. Genet. Evol. 2012, 12, 711-717. [CrossRef]

30. Monteserin, J.; Perez-Lago, L.; Yokobori, N.; Paul, R.; Rodriguez Maus, S.; Simboli, N.; Eldholm, V.; López, B.; de Viedma, D.G.; Ritacco, V. Trends of Two Epidemic Multidrug-Resistant Strains of Mycobacterium tuberculosis in Argentina Disclosed by Tailored Molecular Strategy. Am. J. Trop. Med. Hyg. 2019, 101, 1308-1311. [CrossRef]

31. Belisle, J.T.; Sonnenberg, M.G. Isolation of genomic DNA from mycobacteria. Methods Mol. Biol. 1998, 101, 31-44.

32. Henry, V.J.; Bandrowski, A.E.; Pepin, A.S.; Gonzalez, B.J.; Desfeux, A. OMICtools: An informative directory for multi-omic data analysis. Database 2014, 2014. [CrossRef]

33. Nanoporetechnologies. EPI2ME ARMA Workflow: Real-Time Antimicrobial Resistance Profiling 2020. Available online: https://nanoporetech.com/resource-centre/real-time-detection-antibiotic-resistance-genesusing-oxford-nanopore-technologies (accessed on 28 November 2016).

(C) 2020 by the authors. Licensee MDPI, Basel, Switzerland. This article is an open access article distributed under the terms and conditions of the Creative Commons Attribution (CC BY) license (http://creativecommons.org/licenses/by/4.0/). 